\title{
Polypropylene Laminated Paper (PPLP) Insulation for HVDC Power Cables
}

\author{
George Chen and Zhiqiang Xu \\ The Tony Davies High Voltage Laboratory \\ University of Southampton UK
}

\begin{abstract}
Space charge is a major issue that hinders the development of HVDC polymeric cables. On the other hand, HVDC cables with polypropylene laminated paper (PPLP) insulation has been in operation for a long time with a good record. In the present paper, the electrical performance of PPLP insulation has been studied to understand the reasons for its excellent characteristics. It has been found that the charge accumulated at the interface between PP and Kraft paper plays a key role in determining electrical performance of PPLP insulation. The formation of the interfacial charge results in an optimal electric field distribution for PPLP insulation system as well as a small and electric field independent conductivity.
\end{abstract}

\section{INTRODUCTION}

HVDC power transmission systems have received significant attention as they offer many advantages over HVAC systems in terms of grids connectivity, system reliability and off-shore wind farms etc. As a result, HVDC power cables have experienced a significant development in last ten years. Many efforts have been placed on developing polymeric insulated cables. As a result, insulation based on improved XLPE, nanocomposite and thermoplastic have been developed for HVDC power cables at $400 \mathrm{kV}$ and above [1]. However, less attention has been placed on the insulation based on polypropylene laminated paper (PPLP). PPLP has been widely used for HV power cables including HVDC due to its excellent electrical and mechanical performances. Its use for power cable insulation can be traced back to 1970 's, initially for HVAC cables but extended to HVDC cables and superconducting cables [2-3]. Figure 1 shows a typical PPLP insulated cable structure and PPLP insulation tape.

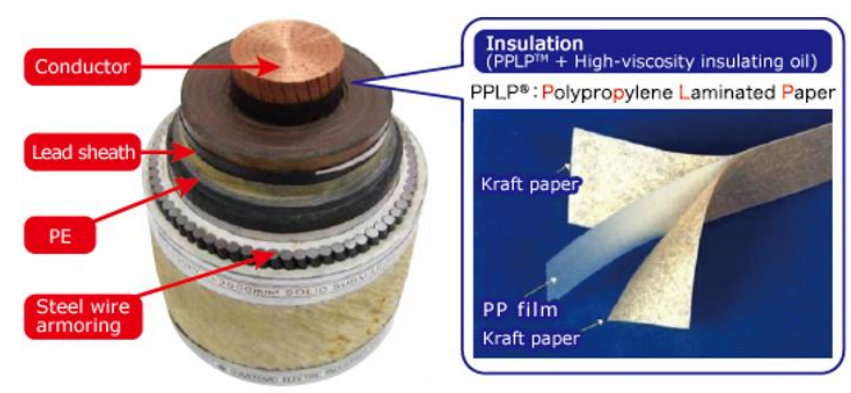

Figure 1 A typical structure of MI HVDC cable and PPLP insulation.
This type of structure shows a higher AC, impulse and DC breakdown strengths as well as lower dielectric loss compared with the conventional Kraft paper insulation. When compared with XLPE insulation the publications on PPLP insulation available in the public domain are limited and the exact mechanisms for the improved electrical performance are not well understood, especially for HVDC cables. Space charge is a major issue that has been highlighted in all insulation for HVDC applications. Therefore, it becomes increasingly important to understand charge dynamics in PPLP insulation system and its influence on the electrical performance of HVDC cables.

In the present paper, charge dynamics in both single and multilayered PPLP films have been studied experimentally using the pulsed electroacoustic technique (PEA). DC electric breakdown and conductivity for PPLP films have also been measured. It has found that the interface between the Kraft paper and polypropylene acts as barrier that hinds the movement of charge carriers. As a result, the electric field distribution in such a system has been significantly modified with the highest electric field in PP and lower electric field in Kraft paper. Similar pattern has been observed in the case of multi-layered PPLP sample while the interface between Kraft papers shows no effect of charge blocking. This type of electric field distribution works favourably for the overall electrical performance of the PPLP insulation system.

\section{SAMPLE PREPARATION AND EXPEREIMENTAL DETAILS}

Sample preparation

PPLP laminated insulation consists of one layer of polypropylene sandwiched between two Kraft paper layers. The samples used in the present study has a total thickness of $\sim 120$ $\mu \mathrm{m}(25 \mu \mathrm{m}+70 \mu \mathrm{m}+25 \mu \mathrm{m})$. The compound used for impregnation is a high viscosity DDB oil (H\&R T2015) which is commercially available. The samples were dried in vacuum oven at $100^{\circ} \mathrm{C}$ before the impregnation process. The DDB oil was degassed at $100^{\circ} \mathrm{C}$ for 24 hours. Impregnation was carried out at $100{ }^{\circ} \mathrm{C}$ in a vacuum oven for 2 days. After being fully impregnated, the samples were kept in vacuum until tests.

\section{Space charge measurement}

Space charge distributions were measured by the pulse electroacoustic technique (PEA). It relies on the interaction of the applied pulsed electric field and the charge in the sample, launching acoustic waves that are detected by a transducer 
attached to one of the electrodes. Through calibration and deconvolution processes, the detected signal can be converted into charge distribution across the sample. To understand charge dynamics in PPLP insulation, single layer Kraft paper and PP were also measured under different electric fields. Typically, the measurements lasted 30 minutes after the external voltage was applied and further 10 minutes after the removal of the external voltage. Since the moisture content is one of the most sensitive factors for oil-paper insulation, the impregnated oil moisture was controlled less than $10 \mathrm{ppm}$. All the experiments were carried out under controlled humidity. The space charge measurements were started after the temperature became stable and data were collected regularly for 30 minutes.

\section{DC electrical breakdown and conductivity measurements}

DC breakdown for Kraft paper, PP and PPLP was measured according to the ASTM D1816. A pair of spherical electrodes were used and the sample was sandwiched between them. Electrodes were rotated after each breakdown to avoid the effect of pitting and replaced after 20 breakdowns. The voltage rise rate is $200 \mathrm{~V} / \mathrm{s}$ and the data were processed using the Weibull statistics.

Electrical conductivity for oil impregnated Kraft paper, PP and oil impregnated PPLP samples was measured for 60 minutes at $20{ }^{\circ} \mathrm{C}$ and $60{ }^{\circ} \mathrm{C}$ under different applied voltages.

\section{RESULTS}

\section{Space charge results}

Figure 2 shows space charge in $50 \mu \mathrm{m}$ Kraft paper impregnated with DDB oil under $20 \mathrm{kV} / \mathrm{mm}$ and $50 \mathrm{kV} / \mathrm{mm}$ at room temperature. Homecharges are observed soon after the application of the external voltage, indicating charge injection taking place from the both electrodes. This is further confirmed by the charge profiles after the removal of the external voltage.

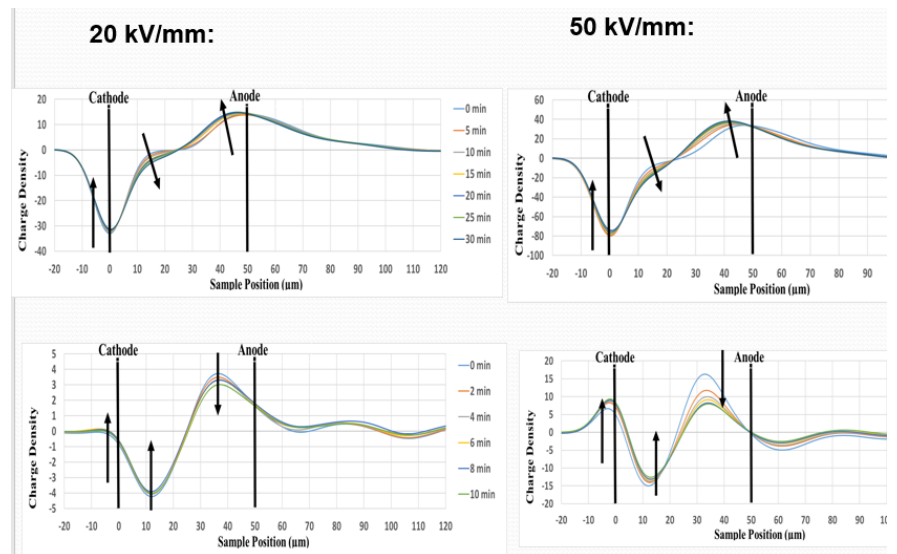

Figure 2 Space charge dynamics in oil impregnated Kraft paper during voltson and decay (room temperature): left $-20 \mathrm{kV} / \mathrm{mm}$ and right $-50 \mathrm{kV} / \mathrm{mm}$.

When the applied electric field increases, more charges are injected as evidenced in Figure 2 (right hand side). Both positive and negative charges are reasonably

balanced/symmetrical under $20 \mathrm{kV} / \mathrm{mm}$ and $50 \mathrm{kV} / \mathrm{mm}$. This observation is more explicit after the removal of the applied electric fields. When the measurement temperature is increased to $60{ }^{\circ} \mathrm{C}$, the movement of charge into the bulk is more obvious, especially for negative charge as shown in Figure 3. Negative charges dominate after the removal of the applied field. It is also believed that more charges may be injected into the sample at higher temperature under the same applied electric field. The charge decay is relatively slow at room temperature but enhanced at $60{ }^{\circ} \mathrm{C}$. These results are consistent with our previous results [4].

$20 \mathrm{kV} / \mathrm{mm}$ :

$50 \mathrm{kV} / \mathrm{mm}$ :
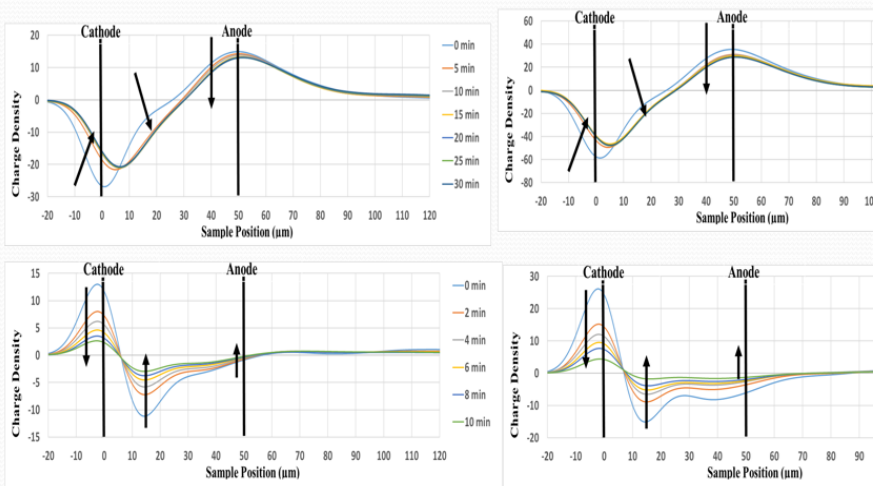

Figure 3 Charge dynamics in oil impregnated Kraft paper during volts-on and decay at $60{ }^{\circ} \mathrm{C}$ : left $-20 \mathrm{kV} / \mathrm{mm}$ and right $-50 \mathrm{kV} / \mathrm{mm}$.

$20 \mathrm{kV} / \mathrm{mm}$ :
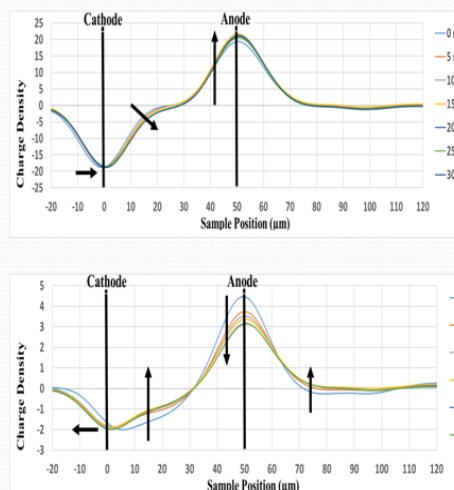

Figure 4 Charge dynamics in oil impregnated PP during volts-on and decay (room temperature): left $-20 \mathrm{kV} / \mathrm{mm}$ and right $-50 \mathrm{kV} / \mathrm{mm}$.

Figure 4 illustrates charge dynamics in $50 \mu \mathrm{m}$ PP film obtained at room temperature at $20 \mathrm{kV} / \mathrm{mm}$ and $50 \mathrm{kV} / \mathrm{mm}$ respectively. It can be seen that the charge injection also occurs at $20 \mathrm{kV} / \mathrm{mm}$ as this electric field is exceeding the threshold electric field and the injection becomes stronger at higher electric field as expected. However, it seems that the negative charge injection plays a crucial role in the charge formation of PP film. Similarly, when increasing the temperature to $60{ }^{\circ} \mathrm{C}$ more charges are injected in the sample, dominated by negative charge under $20 \mathrm{kV} / \mathrm{mm}$ but significant increase in positive charge under $50 \mathrm{kV} / \mathrm{mm}$ as shown in Figure 5 . At $60{ }^{\circ} \mathrm{C}$ the charge buildup in PP film is more obvious and charge decay 
after the removal of the applied field is less fast comparing with that in Kraft paper at the same temperature.

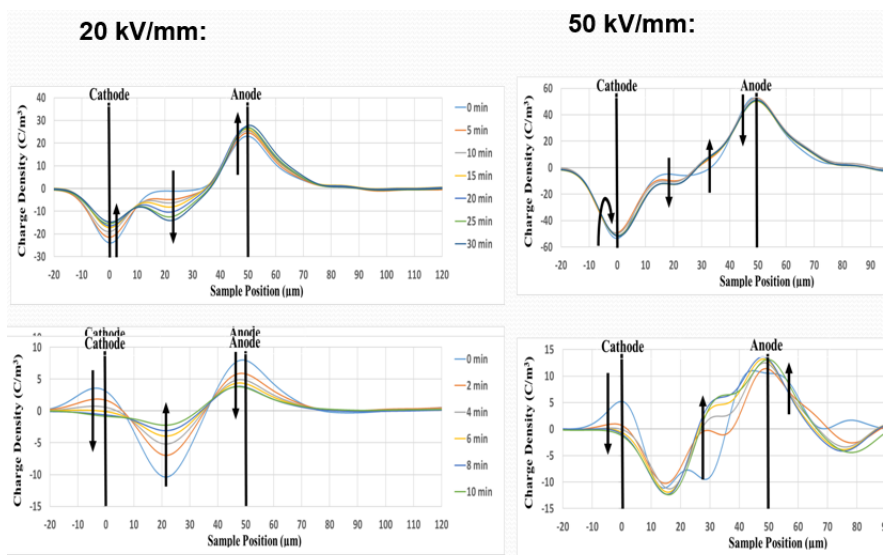

Figure 5 Charge dynamics in oil impregnated PP during volts-on and decay at $60{ }^{\circ} \mathrm{C}$ : left $-20 \mathrm{kV} / \mathrm{mm}$ and right $-50 \mathrm{kV} / \mathrm{mm}$.

Figure 6 shows the charge distribution in PPLP at room temperature. As this is the structure used in HVDC cable, charge dynamics under three electric fields were investigated, i.e. 10,30 and $50 \mathrm{kV} / \mathrm{mm}$. It can be seen that charge reaches to a qusi-steady state at 30 minutes after the application of electric field. Several features can be identified: (i) quick formation of charge at the interfaces (ii) charge at the interface has the same polarity as the closer electrode (iii) the amount of charge at the interface increases with the applied electric field (iv) charge decay after the removal of the field is slow.

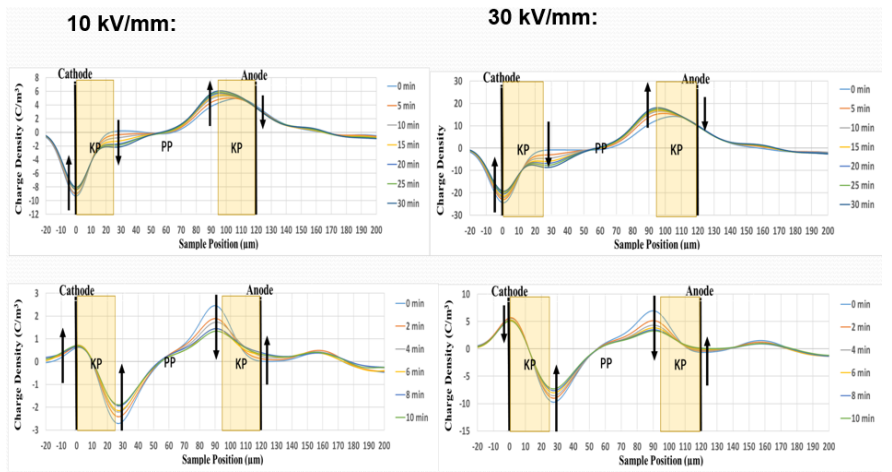
$50 \mathrm{kV} / \mathrm{mm}$ :
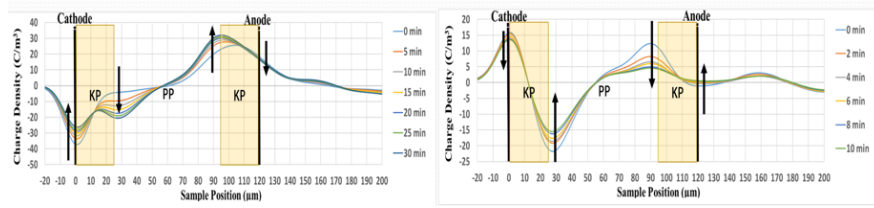

Figure 6 Charge dynamics in PPLP samples at room temperature under 10, 30 and $50 \mathrm{kV} / \mathrm{mm}$.

When increasing the temperature to $60{ }^{\circ} \mathrm{C}$, the buildup of charge at the interfaces is much fast as shown in Figure 7. It seems that the electrodes shift their positions. As a result, the amount of charge at the interfaces is significantly higher than that at room temperature for the same applied electric field. The time required for the interfacial charge buildup decreases with the applied electric field. Higher temperature also enhance charge dissipation from the sample once the applied field is removed.

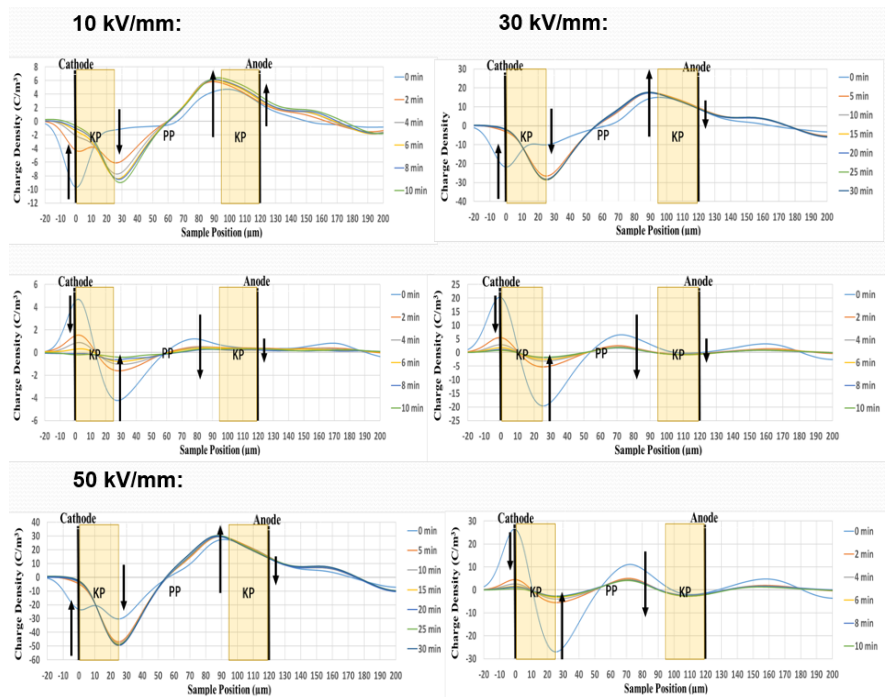

Figure 7 Charge dynamics in PPLP samples at $60{ }^{\circ} \mathrm{C}$ under 10,30 and 50 $\mathrm{kV} / \mathrm{mm}$.

\section{Electrical breakdown strength}

Electric breakdown under dc fields is often related to charge dynamics in the material. Generally, to reduce discharge/flashover, electrical breakdown test is conducted with sample/electrodes immersed in low viscosity silicone oil or transformer oil. However, considering PPLP insulted cable where high viscosity DDB is used, a comparison for PP in both type of oils is made as shown in Figure 8.

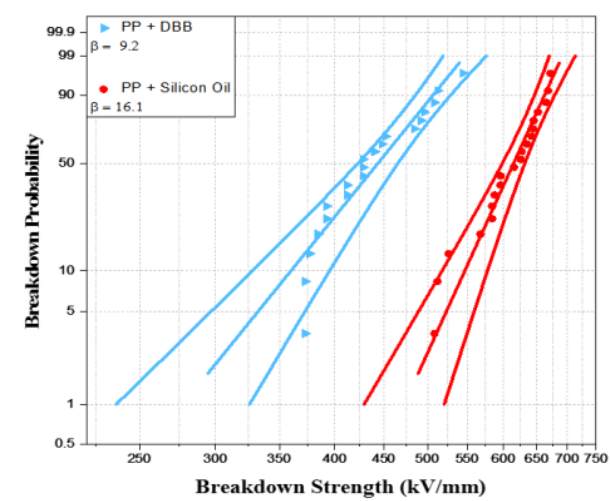

Figure 8 Influence oil on breakdown strength of PP.

It can be seen the breakdown strength of PP in silicone oil is higher than that of DDB. At room temperature, the viscosity of DDB for MI cable is significantly higher than silicone oil, therefore, it is believed that it is extremely difficulty to eliminate pocket cavity. This will lead to a lower breakdown strength as the breakdown voltage is the characteristic of oil/sample system. It is worthy of pointing out that the characteristic breakdown strength $(63.2 \%)$ of PP even in DDB oil is still beyond $450 \mathrm{kV} / \mathrm{mm}$. In reality, PP is laminated between two Kraft papers and the oil impregnation is typically 
carried out in vacuum at high temperature. The probability that voids/cavities can form is much less.

Figure 9 shows the Weibull plot for DDB impregnated Kraft paper and the influence of moisture on the breakdown strength. It is clear that significant impact of moisture on breakdown strength of oil impregnated Kraft paper as expected [5].

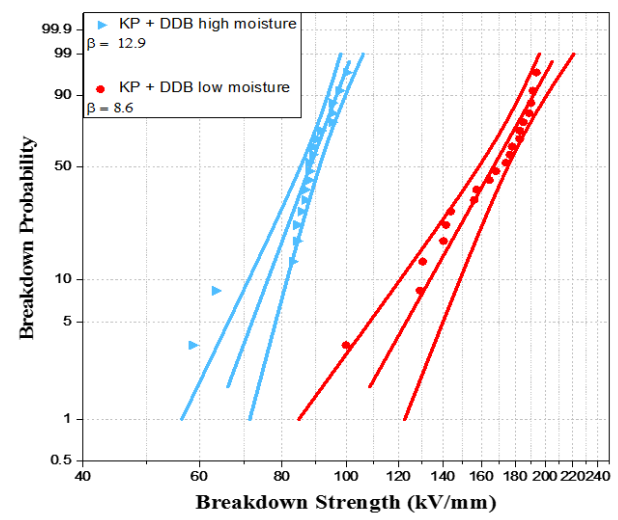

Figure 9 Weibull plot for DDB impregnated Kraft paper and the influence of moisture.

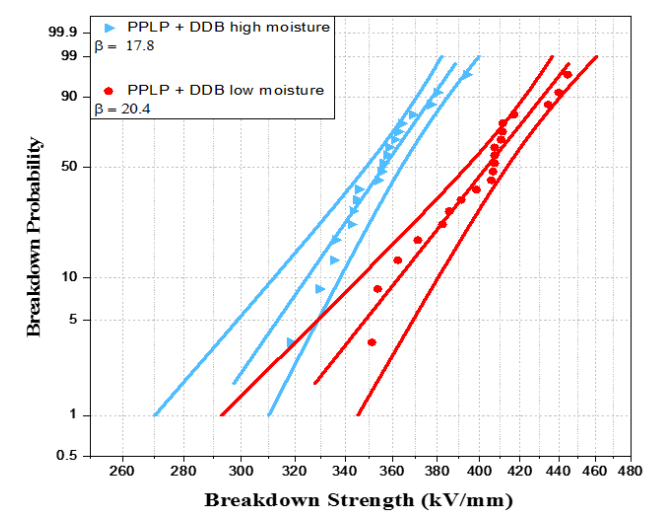

Figure 10 Weibull plot of oil impregnated PPLP and the influence of moisture.

\section{DC conductivity}

DC conductivities for Kraft paper, PP and PPLP were measured at room temperature and $60^{\circ} \mathrm{C}$. The conduction current for both PP and Kraft paper at room temperature is very low. For the sake of clarity, only the conductivity at $60^{\circ} \mathrm{C}$ is shown in Figure 11. Figure 11 (a) illustrates the conductivity change of DDB impregnated Kraft paper with the applied voltages. As expected, the conductivity increases with the voltage (electric field for the fixed ample thickness). Significant increase in conductivity at $5 \mathrm{kV}(50 \mathrm{kV} / \mathrm{mm})$ and the sample failed after just stressing for $20 \mathrm{~min}$. This is not surprising as the electric field applied is above the characteristic breakdown strength of the DDB impregnated Kraft paper. The conductivity of PP film also increases with the applied electric field as shown in Figure 11 (b). However, the conductivity of DDB impregnated PPLP seems independent of the applied electric field as observed in Figure 12 (c). The observation is surprising.

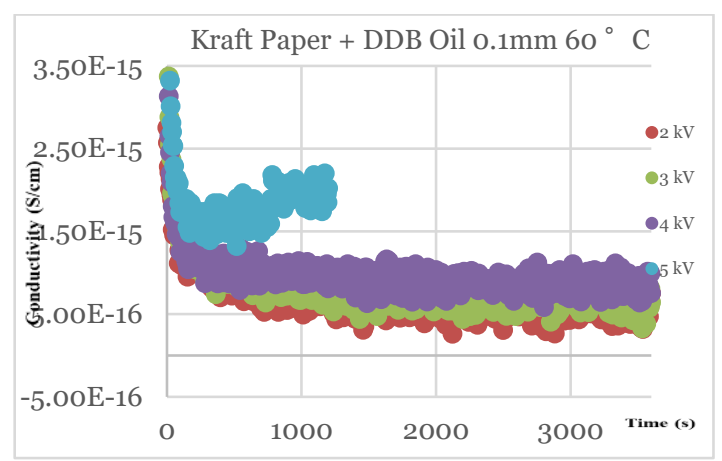

(a)

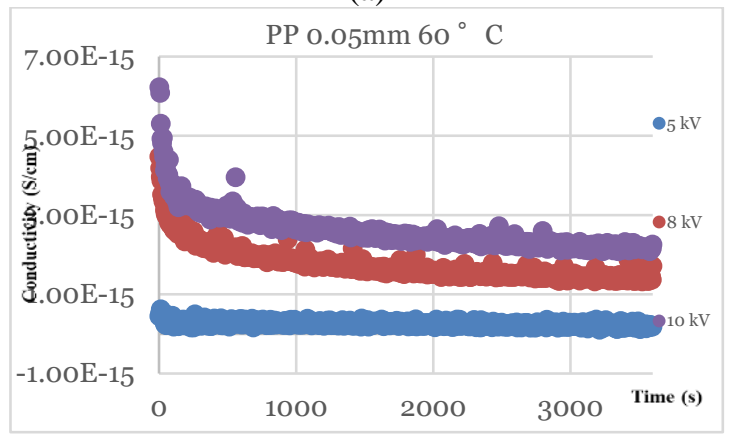

(b)

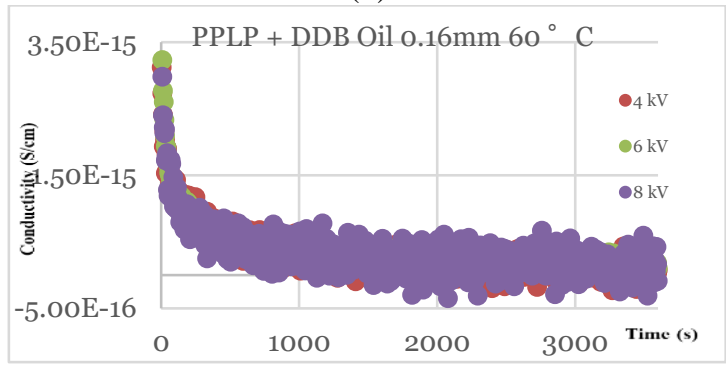

(c)

Figure 11 Conductivity versus the applied voltages.

\section{DISCUSSION}

The presence of charge in the insulation system alters the electric field distribution. Local electric field enhancement may lead to regional material deterioration. This may affect the electrical performance of insulation system and reduce the reliability of high voltage equipment. For the PPLP insulated HVDC cables, the situation is slightly complicated as the electrical breakdown strength Kraft paper is significantly weaker than that of PP film. The uniform electric field distribution is not beneficial for the reliable operation of such insulation system. Therefore, it is important to obtain electric field distribution from space charge measurements. Figure 12 shows the electric field evolution in PPLP over the period of 50 min after application of the voltage. The applied electric field is about $8 \mathrm{kV} / \mathrm{mm}$. After $50 \mathrm{~min}$ the electric field in PP almost doubled while the electric field in Kraft paper decreases significantly. This type electric field distribution is highly desirable as PP is electrically stronger and is able to take extra electric field without deteriorating its electrical performance. 

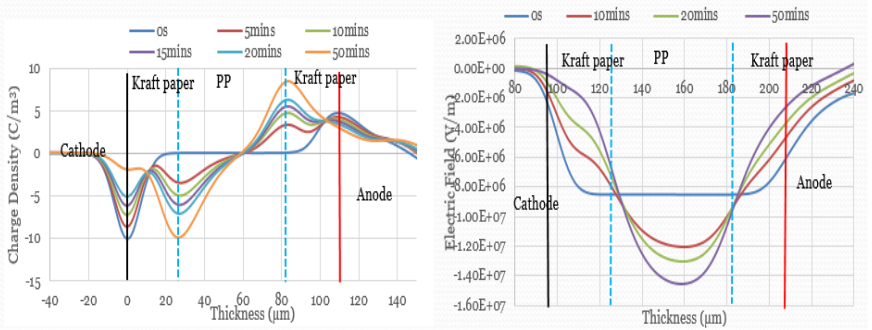

Figure 12 Electric field distribution in PPLP.

Considering the layered structure of the PPLP insulation in real cable. Figure 13 shows charge dynamics and electric field distribution in two-layered PPLP sample at $2 \mathrm{kV}$. Here there are five interfaces within the samples, i.e. four interfaces between Kraft paper and PP and one interface between Kraft papers. It can be seen that the charges are formed only at the interfaces between Kraft paper and PP but not at the interface between Kraft papers. The pattern of charge distribution in each layer PPLP is similar. The electric field distribution calculated based on charge has the same features as those in single layer PPLP. The electric field in PP is enhanced and in Kraft reduced. It seems that such a structure achieves the optimal electric field distribution. The similar results were also obtained for three layered PPLP sample. This may explain why the PPLP insulated HVDC cables shows much better electrical performance than impregnated paper insulated cables.

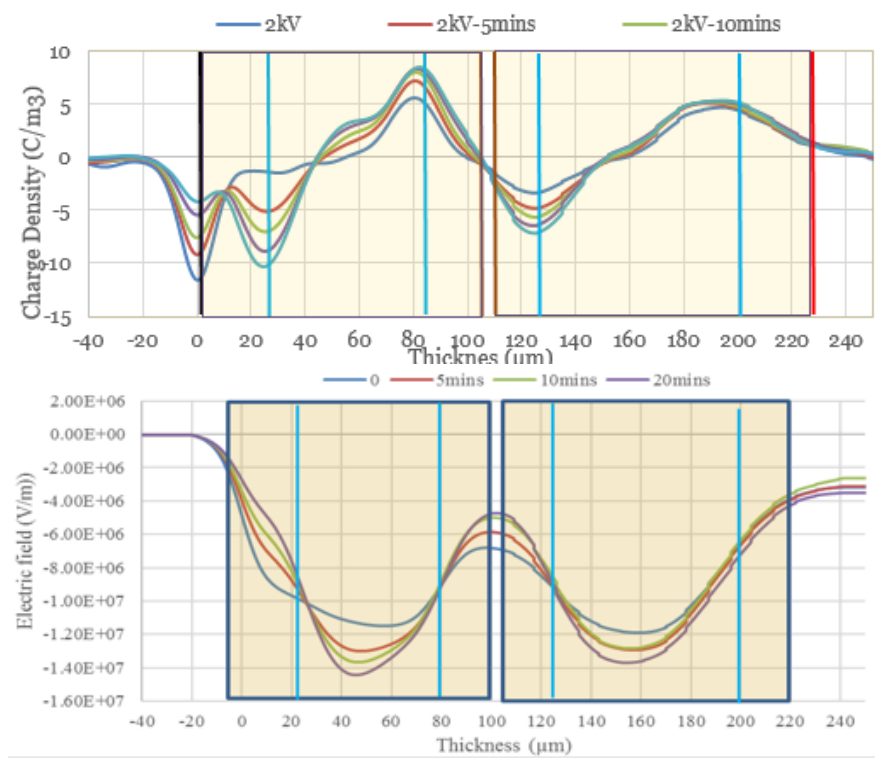

Figure 13 Charge dynamics and electric field distribution in two layered PPLP sample.

In addition, it has been found that PPLP insulation system can also reduce the electrical conductivity as the electric dependent conductivity of Kraft paper is controlled by the lower electric field. Lower electrical conductivity means reduced power loss therefore less heating. The electrical conductivity of PPLP shows little influence by the applied electric field in the range studied as there exists self-modulation between the accumulated charge at the interface and the applied electric field that leads to little change in the electric field in the Kraft paper.

\section{CONSLUSIONS}

Space charge dynamics in Kraft paper and PP have been investigated to understand charge formation in PPLP structure and its electrical performance. The following conclusions may be drawn:

It has been found that the charge injection can easily take place in the impregnated Kraft paper and move fast compared with PP which sees injection at relatively high electric field. The interface between Kraft paper and PP serves as the barrier to charge movement, resulting in the formation of significant amount of charge at the interface. The amount of charge increases with the applied electric field and the movement of the charge increases with temperature.

The direct consequence of the interfacial charges in PPLP system leads to an optimal field distribution with higher electric field in PP and lower in Kraft paper. The pattern of charge and electric field distribution repeat in multi-layered PPLP insulation system. The electrical breakdown strength results show a much better performance of PPLP than Kraft paper.

Another feature observed for PPLP insulation system is that the electrical conductivity shows a small value and little dependent on the applied electric field. This is because the charge at the interfaces adjusts its amount according to the applied electric field and regulates the electric field in the Kraft paper to an almost constant and a small magnitude.

\section{Acknowledgement}

The authors are grateful to the financial support received from UK Engineering and Physical Sciences Research Council (EP/L021560/1 - Towards Enhanced HVDC Cable Systems).

\section{References}

[1] G. Chen, M. Hao, Z. Xu, A. Vaughan, J. Cao, H. Wang, " Review of high voltage direct current cables", CSEE J. Power Energy Systems 2015, 1, 9-21.

[2] A. Fujimori, K. Fujii, H. Takashima, H. Suzuki, M. Mitani, O. Fujii, I. Shigetoshi and M. Shimada, "Development of $500 \mathrm{kV}$ DC PPLP insulated oil-fileed submarine cables", Electr. Eng. in Japan, 1997, 120 (3), 29-41.

[3] I. J. Seo, W. Choi, J. G. Seong, B. W. Lee and J. Y. Koo, " Experimental and analytical study of the DC breakdown characteristics of polypropylene laminated paper with a butt gap condition considering the insulation design of superconducting cable,", Jpn. J. Appl. Phys., 2014, 53, 08 NL04.

[4] B. Huang, M. Hao, J. Fu, Q. Wang, and G. Chen, " Space charge characteristics in oil and oil-impregnated pressboard and electric field distortion after polarity reversal", EEE Trans. Dielectr. Electr. Insul., 2016, 23, 881-891.

[5] R. Liao, J. Hao, G. Chen, Z. Ma and L. Yang, "A comparative study of physicochemical, dielectric and thermal properties of pressboard insulation impregnated with natural ester and mineral oil", IEEE Trans. Dielectr. Electr. Insul., 2011, 18， 1626-1637. 